\title{
Aging in Quenched Poly(methyl methacrylate) under Inelastic Tensile Strain
}

\author{
Shan Zi CUI and Yukuo NANZAI ${ }^{\dagger}$ \\ Department of Mechanical Engineering, Osaka City University, Osaka 558-8585, Japan
}

(Received January 22, 2001; Accepted February 21, 2001)

\begin{abstract}
Aging in quenched poly(methyl methacrylate) (PMMA) under inelastic tensile strain was studied by mechanical test, differential scanning calorimetry (DSC) and thermally stimulated deformation recovery. Evolutions of tensile modulus and yield stress with aging time for PMMA aged under tensile strain were much more significant than those in quenched and physically aged PMMA, giving evidence of aging in quenched PMMA even under negative (expansive) hydrostatic pressure. Experimental data obtained by DSC and the thermally stimulated deformation recovery indicated strain given to the quenched PMMA specimen to be locked into physically aged structure more tightly with aging time.

KEY WORDS Aging / Poly(methyl methacrylate) / Tensile Strain / Expansive Hydrostatic Pressure / Tensile Modulus / Yield Stress / Differential Scanning Calorimetry (DSC) / Deformation Recovery /
\end{abstract}

Commercial products of polymers are used often under strained conditions. A polymeric component tightly fastened by steel bolts and nuts is an illustrative example of such cases. We have so far studied time dependent changes in structure and mechanical properties of noncrystalline polymeric materials given various fixed amounts of strain, comparing with the physical aging behavior of the same materials. ${ }^{1-3}$ As we reported previously, ${ }^{1,3}$ when the samples of quenched poly(methyl methacrylate) (PMMA) and polycarbonate (PC) were aged under compressive strain, their yield stress evolved with aging time much faster than that of physically aged samples (i.e., quenched samples aged with no strain). Aging not in quenched but in fully annealed PMMA samples given inelastic compressive strain was also studied. $^{2}$ This is because quasi-equilibrium polymeric structure made up by full annealing is changed by the imposition of inelastic strain into non-equilibrium structure similar to that of quenched polymer, ${ }^{4}$ thus being expected to reveal some aging behavior under the inelastic strain. As a result, fully annealed and subsequently strained PMMA samples showed the evolution of their yield stress with time much faster than that in physically aged PMMA ${ }^{2}$ hence being similar to the aging in quenched and subsequently strained PMMA samples. Moreover, experimental analysis of strain aging in quenched and fully annealed PMMA samples using differential scanning calorimetry (DSC) and thermally stimulated deformation recovery (TSDR) has led us to the conclusion that the strain given to the sample is locked in physically aged structure more tightly with aging time. ${ }^{1,2}$ Hence, the DSC and TSDR analysis shows that at very long times of aging the amorphous polymer under strain has a structure different from that in physically aged polymer.

In our previous analyses of strain aging in amorphous polymers, ${ }^{1-3}$ samples were strained mostly in compression except in the dynamic viscoelastic analysis of aging in fully annealed PMMA samples strained up to a tensile strain of $0.1 .^{5}$ Hence, in the present paper, we aim to analyze aging in quenched PMMA strained in tension by

\footnotetext{
${ }^{\dagger}$ To whom correspondence should be addressed.
}

means of mechanical test, DSC and TSDR. The analysis leads us consequently to the conclusion that the strain aging already found for compressed samples is also the case for samples strained in tension although they are under the influence of negative (expansive) hydrostatic pressure caused by tensile strain: the elastic modulus and yield stress of quenched PMMA samples strain-aged in tension evolve faster than those in quenched and physically aged samples and the strain is locked into physically aged structure more tightly with aging time.

\section{EXPERIMENTAL}

Dumbbell-shaped specimens with a gage section of 4 $\mathrm{mm}$ in width, $5 \mathrm{~mm}$ in thickness and $40 \mathrm{~mm}$ in length were cut from a commercial cast sheet of PMMA. The glass transition temperature $T_{\mathrm{g}}$ of the sample was determined as $116^{\circ} \mathrm{C}$ by DSC at a heating rate of $1 \mathrm{~K} \mathrm{~min}^{-1}$. Cut specimens were fully annealed at $130^{\circ} \mathrm{C}$ for $2 \mathrm{~h}$. At the end of the annealing process, we cooled the specimens quite rapidly to room temperature by a steady blow of air of room temperature. After quenched by air, specimens were subjected to the physical aging treatment: they were aged in chambers kept at a temperature of $90^{\circ} \mathrm{C}$ for various durations of 1 to $100 \mathrm{~h}$. The aging temperature of $90^{\circ} \mathrm{C}$ is the same as that in the previous study of aging under compressive strain. ${ }^{1}$ After the aging treatment, we moved the quenched and aged specimen (referred hereafter to as specimen QA) to a temperature controlling cabinet of a tensile testing machine and left it for $15 \mathrm{~min}$ at the same temperature as that of the aging treatment. Then we stretched the specimen $\mathrm{QA}$ at a strain rate $\dot{\varepsilon}$ of $6.87 \times 10^{-4} \mathrm{~s}^{-1}$ to have its nominal stress-strain relation.

Specimens for the strain aging, on the other hand, was put into the temperature controlling cabinet kept at $90^{\circ} \mathrm{C}$ of the tensile machine after quenched by air, and left there for $15 \mathrm{~min}$. Then we stretched the specimen to give it a tensile strain of either 0.04 or 0.08 at $\dot{\varepsilon}$ and stopped the tensile machine. Thereafter, we aged the specimen at $90^{\circ} \mathrm{C}$ under the tensile strain for durations of 1 to $100 \mathrm{~h}$, and resume the stretching of the specimen at $\dot{\varepsilon}$ to obtain a nominal stress-strain relation of the 
quenched and strain-aged specimen (referred to as specimen QSA).

For DSC measurements, rectangular parallelepiped DSC specimens of $3 \mathrm{~mm} \times 4 \mathrm{~mm} \times 5 \mathrm{~mm}$ were cut from aged dumbbell-shaped specimens to fit well to the small DSC sample container. The DSC measurement was performed using a DSC 6100 calorimeter of Seiko Instruments and run at a heating rate of $1 \mathrm{~K} \mathrm{~min}^{-1}$ between $-30^{\circ} \mathrm{C}$ and $150^{\circ} \mathrm{C}$.

Thermally stimulated deformation recovery was measured for specimens QSA. The deformation recovery was measured with a laser displacement detector of Keyence Co. at a heating rate of $1 \mathrm{~K} \mathrm{~min}^{-1}$ from $30^{\circ} \mathrm{C}$ to $150^{\circ} \mathrm{C}$.

\section{RESULTS}

\section{Stress-Strain Relations of Aged Specimens}

Control data compared with mechanical behaviors of quenched and strain-aged (QSA) specimens were obtained from quenched and aged (QA) specimens free from strains. The mechanical control data of nominal stress-strain $(\sigma-\varepsilon)$ relations in uniaxial stretching of specimens QA are shown in Figure 1. The yield stress is seen to increase with aging time $t$. In Figure 1, the initial modulus is also seen to increase with aging time though being less obvious than the increase in yield stress.

Nominal stress- ${ }^{-}$strain relations for specimens QSA strained up to 0.04 and 0.08 are shown in Figures 2 and

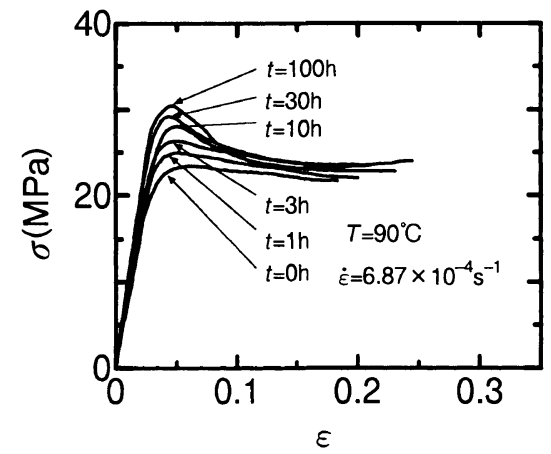

Figure 1. Variation of nominal tensile stress-strain relations with aging time for quenched poly(methyl methacrylate) (PMMA) specimens.

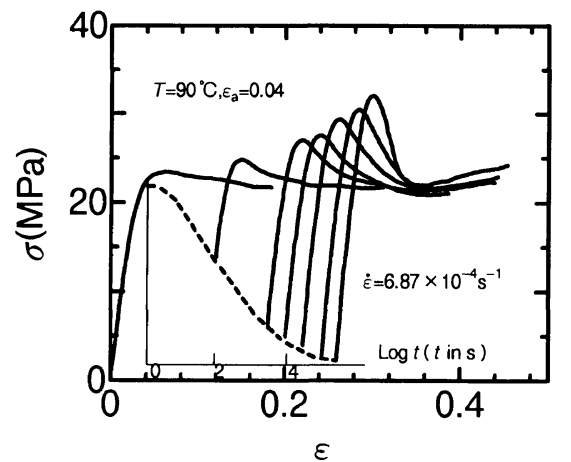

Figure 2. Tensile stress-strain relations for PMMA quenched and aged under tensile strain of 0.04 , showing evolution of yield stress with aging time $t$.
3 , respectively. A horizontal axis of the logarithm of aging time $t$ is inserted to these figures, and the relaxation of tensile stress during the aging time is plotted against $\log t$ with a dashed line. At the end of various aging times, the constant speed uniaxial tension of specimens QSA was run to give nominal stress-strain relations, each of which is drawn in Figures 2 and 3 with a shift of $\log t$ along the axis of nominal strain $\varepsilon$. The yield stress is seen to increase with aging time.

To compare the evolutions of tensile modulus $E$ and yield stress $\sigma_{\mathrm{y}}$ in specimens QA and QSA as a function of aging time, we summarized the tensile modulus and yield stress data of Figures 1, 2, and 3 and plotted them against $\log t$ in Figures 4 and 5, respectively. In Figure 4 , the value of tensile modulus for unaged specimens is given by means of a horizontal dashed line, and filled and open circles are the data for specimens QSA strained up to 0.04 and 0.08 , respectively, while filled triangles represent the control data from specimens QA. The evolution of tensile modulus in specimens QSA is seen to begin much faster than that in specimens QA. For specimens QSA, the slopes of their tensile modulus evolution against $\log t$ are much steeper than that for specimens QA especially at long times. As seen in Figure 4 , the imposed inelastic strain of $\varepsilon_{\mathrm{a}}=0.04$ (a strain before the yield point) and 0.08 (a strain after the yield point) had a quite similar effect on the evolution of tensile modulus. A similar time evolution is also seen for

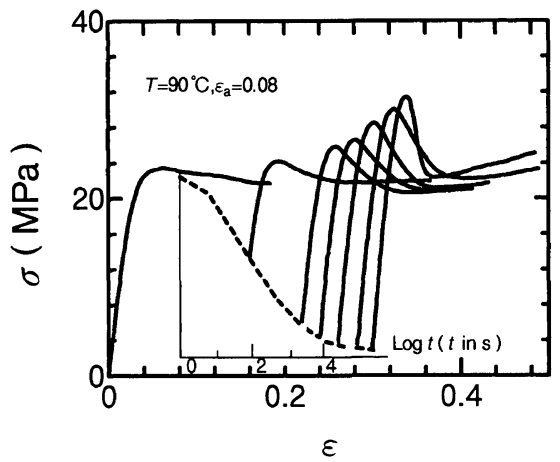

Figure 3. Evolution of yield stress with aging time $t$ for PMMA quenched and aged under tensile strain of 0.08 .

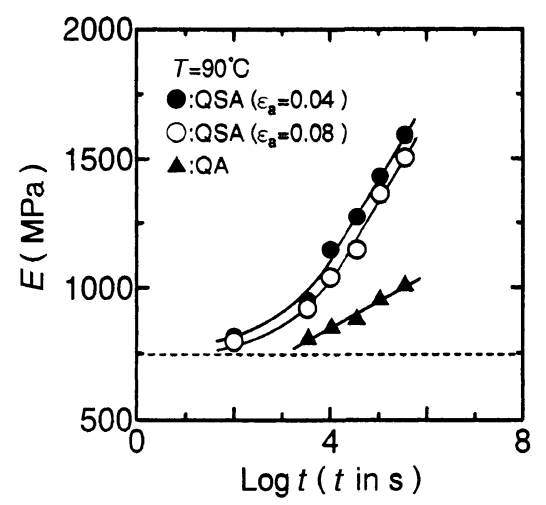

Figure 4. Tensile modulus $E$ plotted against the logarithm of aging time $t$ for three kinds of aged PMMA specimens. , quenched and aged under a tensile strain of 0.04 ; $\bigcirc$, quenched and aged under a tensile strain of $0.08 ; \boldsymbol{\Delta}$, quenched and aged with no strain. 


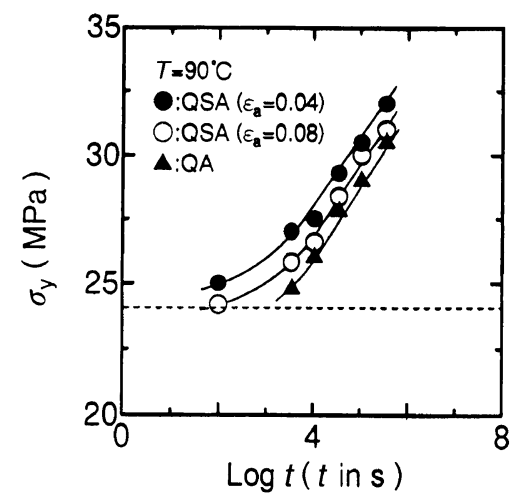

Figure 5. Yield stress $\sigma_{\mathrm{y}}$ plotted against $\log t$ for three kinds of aged PMMA specimens. Symbols are the same as in Figure 4.

yield stress as shown in Figure 5.

\section{DSC Analysis of Aged Samples}

In advance of DSC analysis for aged samples, we examined the effect of machining procedure used to cut small DSC specimens from dumbbell specimens on the DSC data. As a method of examination we made two kinds of DSC samples from a fully annealed PMMA specimen: one is as machined and the other is fully annealed again after the machining procedure. Thermograms for these DSC samples are shown in Figure 6. Little difference is seen between the two DSC curves, thus indicating that the machining procedure has little influence on DSC thermogram.

Thermograms of DSC for specimens QA are shown in Figure 7 as the control data. In the same manner as generally known for quenched amorphous polymers, ${ }^{6}$ the endothermic peak below the glass transition temperature $T_{\mathrm{g}}$ got sharper and shifted to higher temperatures with aging time.

Figure 8 gives the DSC thermograms obtained from specimens QSA strained up to 0.04 and 0.08, and these top and bottom thermograms are corresponding to the stress-strain relations shown in Figures 2 and 3, respectively. In comparison with the thermogram for quenched and unaged PMMA (bold solid line), the endothermic peak developed and showed a shift to higher temperatures with time similarly as seen in Figure 7 for specimens QA. Hence, it may be suggested from Figure 8 that the mechanism of aging in specimens QSA is the same as that in specimens QA. This suggestion can be examined by the comparison of Figure 8 with Figure 7. In Figure 9, we superposed the DSC thermograms of QA and QSA $\left(\varepsilon_{\mathrm{a}}=0.04\right)$ samples obtained at the same aging times upon that of the quenched and unaged sample (thin solid line). Each set of the superposed DSC curves is provided with an arbitrary amount of shift along the vertical axis to avoid a crowd of the curves. As seen by the comparison of the dashed line (QA) with the bold solid line (QSA) at the several aging times, the development of the endothermic peak of specimens QA is always faster than that of specimens QSA. A similar result was also obtained for specimens QSA of $\varepsilon_{\mathrm{a}}=0.08$ as shown in Figure 10.

In consequence, the aging in specimens QSA was shown to be faster than that in specimens QA by the me-

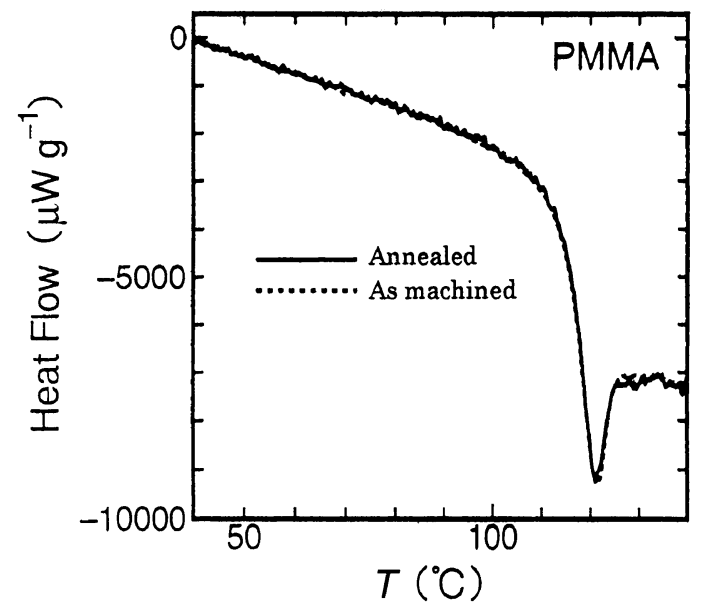

Figure 6. Comparison of differential scanning calorimetry (DSC) thermograms for PMMA specimens as-machined and fully annealed after machining.

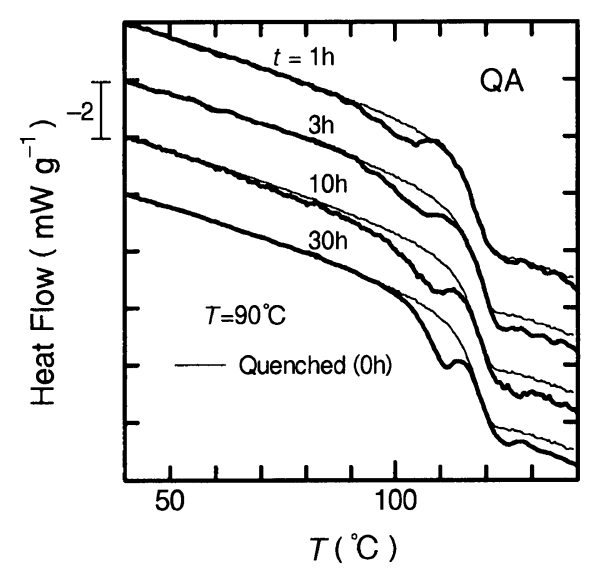

Figure 7. Variation of DSC thermograms with aging time for quenched and aged PMMA.

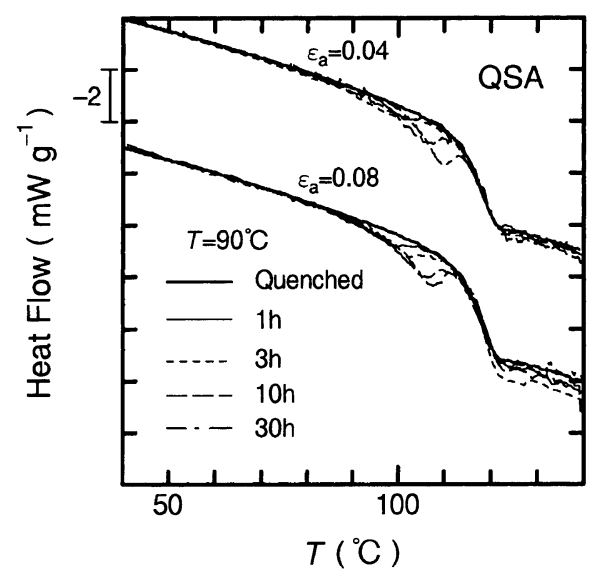

Figure 8. Variation of DSC thermograms with aging time for PMMA quenched and aged under tensile strains of 0.04 (top) and 0.08 (bottom).

chanical experiment, while according to the DSC analysis the aging in specimens QA appeared to be faster than that in specimens QSA. That is, we have contradictory experimental results from mechanical and thermal measurements on the aging in specimens QA and QSA. 


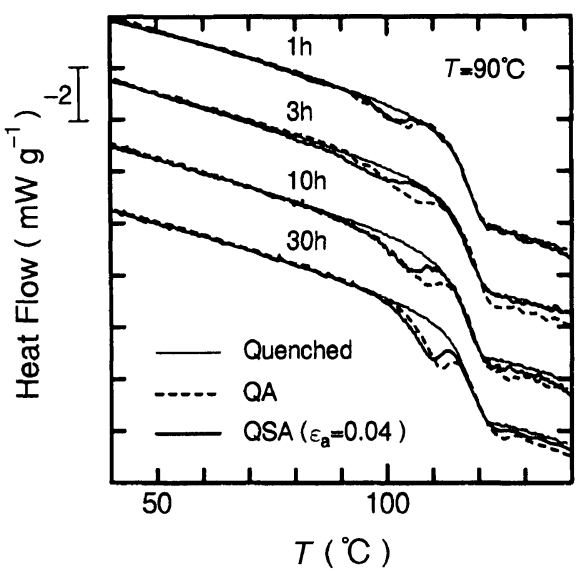

Figure 9. DSC thermograms for quenched PMMA strained up to a tensile strain of 0.04 and then aged (QSA) compared with those for quenched PMMA aged with no strain (QA).

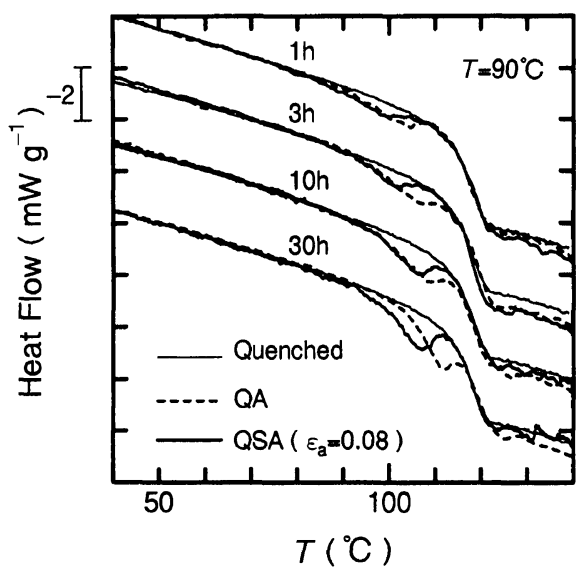

Figure 10. DSC thermograms for quenched PMMA strained up to a tensile strain of 0.08 and then aged (QSA) compared with those for quenched PMMA aged with no strain (QA).

\section{Thermally Stimulated Deformation Recovery of Strain- Aged Samples}

To discuss the contradictory results found above in the mechanical and thermal experimental results of specimens QA and QSA, we must take account of the strain energy stored in the strain-aged specimens and its release at heating. Oleynik ${ }^{7}$ has shown that some amount of strain energy is stored in a finitely deformed amorphous polymer and this is released at the time when the deformed specimen shows deformation recovery at temperatures below $T_{\mathrm{g}}$ at a constant rate of heating. Thus, we accomplished the thermally stimulated deformation recovery experiment on specimens QSA aged for various durations.

Plots of deformation recovery obtained for specimens QSA of $\varepsilon_{\mathrm{a}}=0.04$ and 0.08 are shown in Figures 11a and $11 \mathrm{~b}$, respectively, where a quantity of strain recovery rate $\mathrm{d} \varepsilon / \mathrm{d} T$ is plotted against temperature $T$. The area under the curve gives the total residual plastic strain set in the specimen at the end of the aging process. The recovery peak in the temperature range below $T_{\mathrm{g}}$, where the strain energy is released as reported by Oleynik, ${ }^{7}$ moved to higher temperatures with aging time. We must take into account this energy release (i.e., exothermic
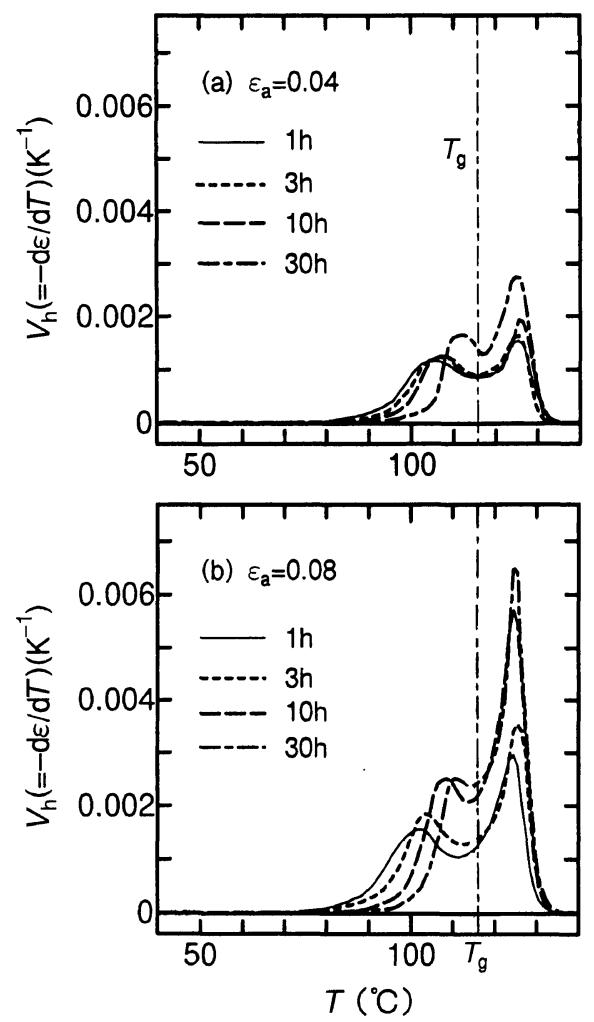

Figure 11. Thermally stimulated deformation recovery for PMMA quenched and aged under tensile strains of 0.04 (top) and 0.08 (bottom)

effect) when examining the DSC result such as shown in Figures 8, 9, and 10. Thus, let us re-examine the DSC thermograms of Figures 9 and 10 by comparing them closely with the deformation recovery shown in Figure 11. The plot for the comparison is shown in Figure 12 for $\varepsilon_{\mathrm{a}}=0.04$. Each of arrows on the bottom panel points to the peak of sub- $T_{\mathrm{g}}$ deformation recovery. The strain energy is expected to have been released around the temperature pointed by each arrow. In the same temperature range of the arrow on the top panel, say for $t=30 \mathrm{~h}$, the endothermic curve is depressed towards the exothermic direction, probably because of the thermal energy balance between the endothermic behavior caused by an aged structure brought by ordinary physical aging and the exothermic effect due to the release of strain energy. Similar depressions of the endothermic behavior are seen also in the cases of $t=1,3$, and $10 \mathrm{~h}$ in a range around the temperature pointed by each arrow. Endothermic behavior due to the structure brought by ordinary physical aging began to appear first, and then at slightly higher temperatures (around each arrow) the strain energy was presumably released simultaneously with the endothermic behavior. Thus, a transient part of the thermogram being first endothermic and then depressed towards the exothermic direction indicates that the strain energy has been locked into the molecular structure stabilized by ordinary physical aging. That is, the strain energy could be released after the stabilized structure locking that strain energy in itself had been loosened by the thermal energy absorption.

The relation between DSC thermograms and deformation recovery curves similar to that in Figure 12 is also 


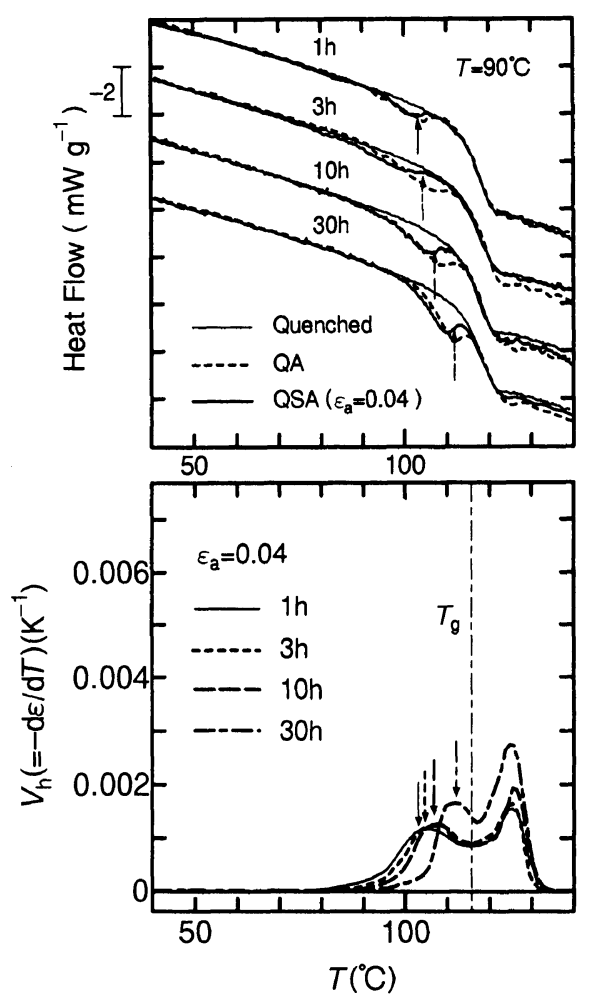

Figure 12. Deformation recovery curves (bottom) compared to DSC thermograms (top) for PMMA quenched and aged under tensile strain of 0.04 and PMMA quenched and aged with no strain.

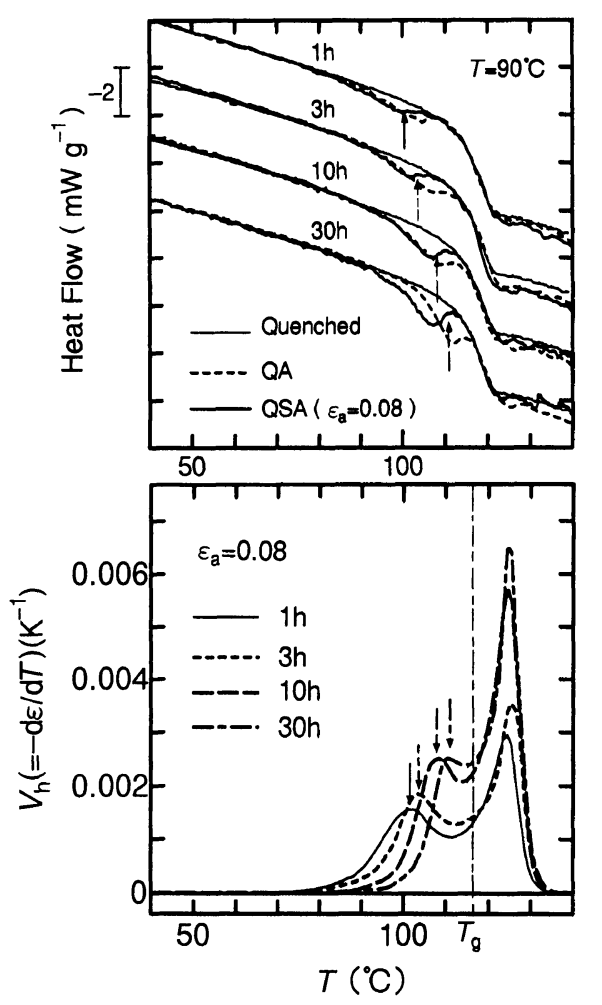

Figure 13. Deformation recovery curve (bottom) compared to DSC thermograms (top) for PMMA quenched and aged under tensile strain of 0.08 and PMMA quenched and aged with no strain.

seen for $\varepsilon_{\mathrm{a}}=0.08$ as shown in Figure 13.

\section{DISCUSSION}

The steady shift of the sub- $T_{\mathrm{g}}$ recovery peak to higher temperatures with aging time shown in Figure 11 indicates that the strain energy comes to be locked in the stabilized (physically aged) structure more tightly with aging time. Since the yield stress increased simultaneously with the shift of the sub- $T_{\mathrm{g}}$ recovery peak to higher temperatures, we can conclude that the stabilized structure locking the strain energy in itself contributes to the significant increase in yield stress.

On the DSC thermograms shown in Figures 9 and 10 for specimens QA and QSA, we should notice that the tail of endothermic peak of specimens QSA is quantitatively in excess of that of specimens QA on the low temperature side. This probably implies that the approach to the thermodynamically most stable structure (i.e., physical aging) in specimens QSA is not so much attained as in specimens QA. Yet, as seen in Figures 4 and 5 , the aging in specimens QSA is much faster than that in specimens QA in terms of tensile modulus and yield stress. Hence, the evolution of the tensile modulus and yield stress in specimens QSA faster than that in specimens QA is ascribable to the structure locking the strain energy in itself. This structure supposedly has a property quite effective to resist against mechanical deformation.

The key finding in the present study is the experimental evidence of strain aging in quenched PMMA under inelastic tensile strain, which is quite similar to the strain aging under inelastic compressive strain reported previously. That is, the strain aging was shown to evolve even under negative (expansive) hydrostatic pressure caused by tensile strain. As we have mentioned above, the strain aging is a relaxation process where the strain is locked into the physically aged structure more tightly with aging time. Thus, physically aged structure in strained PMMA was shown to evolve even under negative hydrostatic pressure, suggesting strongly that physical aging in quenched polymers with no strain is also probable under the condition of negative hydrostatic pressure.

\section{CONCLUSIONS}

Mechanical and thermal analysis was performed on aging in quenched PMMA under inelastic tensile strain. Evolutions of tensile modulus and yield stress with aging time for PMMA aged under tensile strain were much more significant than those in quenched and physically aged PMMA, thus giving evidence of aging in quenched PMMA even under negative (expansive) hydrostatic pressure. Experimental data obtained by DSC and the thermally stimulated deformation recovery indicated strain given to the quenched PMMA specimen to be locked into physically aged structure more tightly with aging time.

\section{REFERENCES}

1. Y. Nanzai, A. Miwa, and S. Z. Cui, JSME Intl. J. Series A, 42, 479 (1999). 
2. Y. Nanzai, A. Miwa, and S. Z. Cui, Polym. J., 32, 51 (2000).

3. S. Z. Cui, Y. Nanzai, and S. Yoshioka, Kobunshi Ronbunshu, 57, 37 (2000).

4. Y. Nanzai, Prog. Polym. Sci., 18, 437 (1993).

5. S. Yoshioka, H. Usada, and Y. Nanzai, J. Non-Cryst. Solids,
172-174, 765 (1994)

6. L. C. E. Struik, "Physical Aging in Amorphous Polymers and Other Materials", Elsevier Science Publishers, Amsterdam, 1978.

7. E. Oleynik, Prog. Colloid Polym. Sci., 80, 140 (1989). 